\title{
Reflexões sobre o Diabetes Tipo 1 e sua Relação com o Emocional
}

\author{
Daniela Botti Marcelino' \\ Maria Dalva de Barros Carvalho \\ Universidade Estadual de Maringá
}

\begin{abstract}
Resumo
Este artigo tem como tema central o diabetes tipo 1 ou infanto-juvenil. Tendo em vista as repercussões que esta doença crônica traz, este trabalho levantou dados bibliográficos que subsidiassem reflexões sobre os aspectos emocionais vivenciados pelas crianças diabéticas. $\mathrm{O}$ resultado mostrou que o emocional influi na etiologia do diabetes e que o diabetes também influi no emocional desta criança. Isso ocorre devido aos cuidados incessantes para controle da doença, medo das possíveis complicações, mal-estar provocado pelos sintomas de hiper e hipoglicemia, o fato de ser "diferente". Sendo assim, é necessário um acompanhamento desta criança não só por médicos, mas também por outros profissionais da saúde, entre eles o psicólogo, objetivando elaboração dos aspectos referentes à doença e suas repercussões emocionais. Palavras-chave: Diabetes mellitus tipo 1; educação acerca do diabetes; aspectos emocionais; psicoterapia de grupo.
\end{abstract}

Reflections on the Diabetes Type 1 and its Relation to the Emotional Aspect

\begin{abstract}
This article has as its main point the type 1 or infantile-juvenile diabetes. Considering the repercussions that this chronic disease may cause, this study aims at performing a bibliographic survey with data that can give a subsidy to reflections on the emotional aspects experienced by the diabetic children. The findings showed that the emotional aspect influences the diabetes etiology and that the diabetes also influences the emotional aspect of this child. This is due to both the incessant cares used to control the disease and fear of possible complications, as well as to the indisposition caused by the hyper and hypoglycemia symptoms and the fact of being "different". Therefore, it is necessary an attendance of these children diabetes, not only by the doctors, but also by other health professionals, among them, the psychologist, aiming at the elaboration of the aspects related to the disease and its emotional repercussions.

Keywords: Diabetes mellitus type 1; education about diabetes; emotional aspects, psychotherapy in group.
\end{abstract}

O primeiro caso de diabetes foi constatado no Egito em 1500 a.C., como uma doença desconhecida. A denominação diabetes foi usada pela primeira vez por Apolonio e Memphis em 250 a.C. Diabetes em grego quer dizer sifão (tubo para aspirar a água), este nome foi dado devido a sintomatologia da doença que provoca sede intensa e grande quantidade de urina. $O$ diabetes só adquire a terminologia mellitus no século I d.C.; Mellitus, em latim, significa mel, logo a patologia passa a ser chamada de urina doce. (Gama, 2002).

O diabetes mellitus é uma doença crônica, caracterizada pela elevação da glicose (açúcar) no sangue acima da taxa normal (hiperglicemia). A taxa normal é de aproximadamente 60 a $110 \mathrm{mg} \%$. Ele é causado por fatores genéticos (herdados) e ambientais, isto é: a pessoa quando nasce já traz consigo a possibilidade de ficar diabética. Quando, aliado a isso, se traz fatores como obesidade, infecções bacterianas e viróticas, traumas emocionais, gravidez etc., a doença pode surgir mais cedo. (Zagury, Zagury \& Guidacci, 2000, p. 16)

Segundo Zagury e colaboradores (2000), o diabetes pode ser causado também por: cirurgias, estresse, alimentação rica em carboidratos concentrados como balas, doces, açúcar, menopausa e certos medicamentos. Os principais sintomas do diabético são: muita sede (polidipsia), excesso de urina (poliúria), muita fome (polifagia), e emagrecimento. Outros sintomas são: sonolência, dores generalizadas, formigamentos e dormências,

\footnotetext{
${ }^{1}$ Endereço para correspondência: Rua Distrito Federal, 50. Jd. Novo Horizonte, Maringá, PR, 87005 100. Fone: (44) 32266172. E-mail: dani_botti@yahoo.com.br
}

cansaço doloroso nas pernas, câimbras, nervosismo, indisposição para o trabalho, desânimo, turvação da visão, cansaço físico e mental.

Se não houver um bom controle da doença podem surgir complicações como problemas de visão (retinopatia), problemas nos rins (nefropatia) e problemas neurológicos (neuropatia). As complicações a nível cerebral ocorrem porque "o cérebro depende da glicose pela demanda excessiva de energia que as funções cerebrais necessitam" (Grünspun, 1980, p. 407). Pode-se notar distúrbios como: cefaléia, inquietude, irritabilidade, palidez, sudorese, taquicardia, confusões mentais, desmaios, convulsões e até o coma.

Segundo as estimativas e projeções mundiais do diabetes mellitus apresentadas por McCarty e Zimmet (1994), em 2000 teríamos 175 milhões de diabéticos em todo o mundo e em 2010 este índice aumentaria para 236 milhões, no Brasil as estimativas seriam de 5 milhões em 2000 e 11 milhões em 2010. Há vários estudos verificando estes índices de prevalência e incidência do diabetes, um deles é o Projeto Multinacional para o Diabetes na Infância (DIAMOND), que vem constatando um aumento da frequêencia do diabetes tipo 1 (Delamater \& cols., 2001; GEED - Grupo de Estudos em Endocrinologia \& Diabetes, 2001). Campanhas nacionais do Ministério da Saúde realizaram, em 2001, 21 milhões de glicemias capilares e detectaram 3 milhões de suspeitos de diabetes, o que equivale a 7\% da população pesquisad. (Brasil, 2002).

Há basicamente dois tipos de diabetes: tipo 1 e tipo 2. Além de suas diferenças na sintomatologia e tratamento, elas se diferenciam na população que atingem. $\mathrm{O}$ diabetes tipo 1 atinge crianças e adolescentes, já o tipo 2 atinge principalmente a população entre $30 \mathrm{e}$ 69 anos, embora hoje já se observe este quadro também em crianças devido a obesidade e ao sedentarismo infantil (Delamater \& cols., 2001; Graça, Burd \& Mello Filho, 2000; Zagury \& cols., 2000). 
Como as estimativas mostram, o diabetes é uma doença que atinge milhões de pessoas e que tende a crescer, ou seja, há uma grande população que enfrenta uma doença crônica e incurável que está se alastrando (GEED, 2001). Sendo uma doença incurável é preciso investir no diagnóstico precoce e em seu tratamento para obtenção da melhor qualidade de vida possível ao seu portador. Sabe-se que grande parte dos novos casos de diabetes tipo 2 poderiam ser prevenidos evitando o excesso de peso e combatendo o sedentarismo. Já nos casos do tipo 1 ainda não há medidas de prevenção da doença, mas pode-se prevenir as complicações crônicas decorrentes dela (Diabetes, 2003).

A medicina sozinha não consegue dar conta de ajudar no controle da doença para que não haja complicações, pois além de exames e medicamentos, é necessário dieta, exercícios físicos e um equilíbrio emocional, visto que estes aspectos influem diretamente sobre a doença, descontrolando-a e agravando o quadro (GEED, 2000). Pode-se observar, por exemplo, casos em que o paciente tem consciência da importância de um bom controle sobre a doença, das conseqüências de um mal controle, mas, ainda assim, não se ajuda e burla o tratamento. Isto ocorre porque não basta ter consciência da doença e suas repercussões, pois a doença física atinge diretamente o emocional e este não é determinado apenas por aspectos conscientes. $\mathrm{O}$ emocional é constituído por aspectos mais profundos internamente e inconscientes, que podem impedir um bom controle da doença se esta não for internamente aceita. Debray (1995) acredita que o diabetes será enfrentado diferentemente por cada indivíduo, pois dependerá da estrutura psíquica ou organização mental de cada um.

Diante do exposto é necessário refletir a respeito da influência dos aspectos emocionais sobre o diabetes tipo 1 e a importância de um atendimento psicológico para seus portadores. É o que se propõe este trabalho com um levantamento de dados bibliográficos a partir de termos como diabetes mellitus, aspectos emocionais relacionados ao diabetes, diabetes tipo 1 ou infantojuvenil, psicoterapia de grupo e diabéticos, psicossomática, controle glicêmico e os mesmos termos também em inglês. $\mathrm{O}$ levantamento bibliográfico foi realizado no período de junho de 2002 a agosto de 2003 em diversos meios como livros, Data Capes e outros sites da internet, artigos em periódicos e outras revistas, abstracts de eventos científicos, etc.

\section{Desenvolvimento}

Vários autores, entre eles Graça e colaboradores (2000), Grünspun (1980), Anjos (1982), Ajuriaguerra (1976), Joode (1976) e Debray (1994), consideram o diabetes mellitus uma doença psicossomática, ou seja, que sofre influência de fatores emocionais em sua etiologia.

Silva (1994) afirma que doença psicossomática é qualquer alteração somática (física) decorrente de sofrimentos psíquicos, diferentemente da somatopsíquica que é qualquer alteração psíquica decorrente de sofrimento físico, por exemplo, os efeitos psíquicos sofridos pelo indivíduo que possui uma enfermidade crônica ou uma debilidade física. Sendo assim o diabetes mellitus pode ser considerado tanto uma doença psicossomática quanto somatopsíquica.

Para Silva (1994), a doença psicossomática surge em decorrência do modo como o indivíduo vivencia as emoções. As emoções (medo, ira, amor) são situações novas frente às quais o organismo se desequilibra e se prepara para descarregá-las através dos músculos voluntários do corpo. Porém, muitas vezes, as emoções não são descarregadas, expressas, talvez pela imposição da sociedade para reprimi-las. Quando as emoções não são expressas através de nossos músculos voluntários, elas descarregam-se em nossos músculos involuntários, como o estômago, intestino, coração e vasos sangüíneos, podendo desencadear a doença psicossomática. “... carregada de agressividade contida, a pessoa não agride os outros mas a si mesmo..." (p. 167). A melhor maneira de se descarregar a emoção é através do choro convulsivo; outra forma também valiosa é através das palavras.

A incapacidade de comunicar com palavras os seus pensamentos faz com que essa pessoa "fale" com a "linguagem dos órgãos", ou seja, o adoecer de determinado órgão é a forma inconsciente do indivíduo proclamar seu sofrimento, por não conseguir fazê-lo de outra forma... (Silva, 1994, p. 87)

Não quer dizer que Silva (1994) considere que todas as doenças são causadas unicamente por aspectos emocionais, ele considera que a doença decorre de vários fatores (pluricausalidade), causas internas e externas e inclui o emocional nesta pluricausalidade.

Tanto Debray (1995), como Anjos (1982), parecem concordar com Silva (1994), quando afirmam que o diabetes mellitus é uma doença multifatorial, ou seja, pode ser decorrente de vários fatores.

Grünspun (1980) também apresenta uma visão similar aos autores acima, pois afirma que "entre os diferentes quadros clínicos dos distúrbios do sistema endócrino, o diabetes mellitus na criança tem sido considerado com freqüência, como distúrbio psicossomático" (p. 414). Debray (1994) e Anjos (1982) acreditam que o diabetes se desencadeia principalmente por fatores hereditários, mas só o fator hereditário não é suficiente para que haja a doença, são necessárias “... modificações exteriores violentas com valor de trauma ...” (Debray, 1994, pp. 25-26). Ela também afirma que o trauma, por si só, não seria responsável pelo aparecimento da doença, logo o diabetes é multifatorial.

Na anamnese de adolescentes diabéticos, E.P.Stein e V.Charles (1971) verificaram perdas parentais (morte, separação, divórcio) ou distúrbios familiares graves em uma proporção significativamente elevada, o que, segundo eles, justifica a hipótese de que um indivíduo fisiologicamente sensível, em um clima de estresse afetivo, é mais susceptível a desenvolver manifestações clínicas do diabetes ... (Ajuriaguerra, 1976, p. 838)

Anjos (1982) também considera os problemas afetivos envolvendo os pais e a família e até problemas escolares como significativo no surgimento do diabetes.

Estudos recentes tem mostrado que depressão constitui um fator de risco maior para desenvolvimento do diabetes e de suas 
complicações. Além disso, tratamentos a curto prazo para depressão em diabéticos melhora a disforia e outros sinais e sintomas da depressão (Musselman, Betan, Larsen \& Phillips, 2003).

Correlacionando o quadro da doença com os aspectos emocionais envolvidos no diabetes, como as perdas citadas anteriormente, pode-se fazer uma análise simbólica. O indivíduo que vivencia uma perda, sofre por ela, sente raiva do objeto perdido, tem o desejo de jamais ter amado, pois assim não teria sofrido com a perda. O desejar não amar para não sofrer, segundo Silva (1994), é um sentimento comum entre as pessoas que somatizam, ele classifica isso como narcisismo - o não conseguir amar o outro por não tolerar a frustração de perdê-lo. Toda esta dinâmica psíquica envolvida na perda, se assemelha ao quadro do diabetes, pois o diabético não permite que o açúcar (doce), que simboliza amor, entre nas suas células, ou seja, não permite que o amor entre no seu corpo e o faça sofrer. $\mathrm{O}$ diabético transporta o emocional (medo de sofrer) para o soma (não permitir a entrada do açúcar, simbolicamente do amor). Isso também pode ser observado na alteração da glicemia. Segundo Vale, Ornellas e Franco (1979), quando o diabético vive uma tensão emocional há um aumento de glicemia, assim quando o diabético fica tenso permite menos ainda que o açúcar (doce) entre na célula.

Segundo GEED (2000, pp. 43-44) a doença interfere na capacidade de amar:

Dependendo de como a doença se manifesta e do significado que ela (a doença) tem na fantasia do paciente, pode atingir o seu "eu", a sua auto-estima, a qualidade de vida, a capacidade de amar, de ter esperanças, as possibilidades e qualidades de relações pessoais. A doença, seja ela sofrida ou implicando em mudanças no estilo de vida, nunca é bem recebida. Mesmo os que parecem mais adaptados têm uma dose de revolta por trás de sua tolerância, porque ninguém quer ficar doente.

Os sentimentos que acompanham o adoecer são negativos, pois as pessoas ao depararem com algo diferente e invasivo, demonstram sentimentos de menos valia, inferioridade, medo, raiva, ansiedade e até depressão.

Outros autores também abordam a respeito dos sentimentos vivenciados pelos pacientes diabéticos. Silva (1994), por exemplo, acredita que o adoecer produz um forte agravo na auto-estima do indivíduo.

Menninger e Daniels (1976, citados em Grünspun, 1980) constataram a presença da depressão e da ansiedade na maioria dos diabéticos. Grünspun afirma que há um agravamento dos distúrbios das glândulas endócrinas quando o indivíduo sofre alterações emocionais, principalmente depressão. Ele acredita tanto no caráter emocional do diabetes que chega a afirmar a existência de casos de diabetes que foram controlados sem medicamento, apenas com tratamento para depressão. Zagury e colaboradores (2000) discordam que o tratamento do diabetes possa funcionar sem medicamento e afirmam que o controle depende da aprendizagem sobre a doença, da dieta, do uso de insulina, etc.

Para Silva (1994) os pacientes diabéticos têm sentimentos de inferioridade devido aos cuidados constantes exigidos para controlar a doença. Ajuriaguerra (1976) também concorda que o controle do diabetes é muito intenso e chega a classificar a criança diabética como escrava de seu tratamento. Debray (1994) concorda com Ajuriaguerra quando define o diabetes como "... uma doença irreversível que exige um tratamento médico severo e contínuo..." (p. 26).

Debray (1994) relata que um adolescente de 17 anos apresentou ambivalência entre as exigências superegóicas e o desejo de desprender-se delas. Esta ambivalência pode estar ligada ao tratamento severo e contínuo ao qual o diabético tem que se submeter. Hartmann, Dellatorre e Marcelino (2002) percebem esta ambivalência vivenciada por crianças e adolescentes diabéticos em um grupo psicoterapêutico:

Outro aspecto apresentado por todos os participantes do grupo foi
o conflito entre a impulsividade versus o controle intelectual e
superegóico. Este traço de personalidade está muito ligado às
características da doença, pois o paciente diabético tem que estar
sempre se fiscalizando, controlando a alimentação, a contagem de
carboidratos, a glicemia, a aplicação de insulina. Uma criança, durante
um dos encontros, fez um desabafo a este respeito dizendo que não
podia ficar nervoso, porque sua glicemia já se descontrolava e ela
começava a passar mal. (p. 42)

Tanto Ajuriaguerra (1976), quanto Vale e colaboradores (1979) parecem fundamentar o desabafo desta criança quando afirmam que as modificações na glicemia e na evolução da doença de maneira geral, pode ser causada por fatores emocionais e afetivos. Graça e colaboradores (2000, p. 221) cita a fala de uma das participantes de um grupo de diabéticos que confirma esta posição “...'se não controlar a emoção, não controla a doença'..."

Segundo Joode (1976), o paciente diabético apresenta sentimento de inferioridade e inadequação em decorrência dos distúrbios no desenvolvimento físico como baixa estatura e desenvolvimento sexual retardado. Joode afirma também que os diabéticos apresentam ansiedade a respeito da saúde, medo da morte e idéias de suicídio.

Silva (1994) cita o caso de um jovem diabético que vivia um casamento insatisfatório e infeliz e foi encontrado morto “...vítima de um excesso de insulina, num provável ato suicida inconsciente"(p.118).

Bleger (1963, citado em Ajuriaguerra, 1976) também aborda estas questões “...considera que as tendências depressivas são freqüentes e que a maior parte dos jovens diabéticos apresenta ansiedade, angústia e inquietação consciente diante do futuro, e esta angústia pode gerar idéias de suicídio...” (p. 839).

Graça e colaboradores (2000, p. 226) citam o depoimento de dois pré adolescentes durante sessões de grupo: "Minha mãe diz que sou chata, mas eu nem ligo, reclamo mesmo. Dieta é muito ruim. Sabe o que eu falo? Que quero morrer e nem precisa caixão para me enterrar. É só embrulhar no jornal..."; “Seria bom se eu morresse. Aí, eu nascia de novo e podia comer de tudo, enquanto rabisca um papel com um lápis vermelho."

Ajuriaguerra (1976) acredita que antes dos 10 anos de idade o paciente diabético aceita a doença mais passivamente, sem ter muita consciência dela, mas com a adolescência chega também a revolta e a dissimulação. Já Graça e colaboradores (2000) 
parecem relacionar a questão da aceitação da doença com os recursos internos e não especificamente com a fase de desenvolvimento em que o paciente se encontra.

Pesquisas indicam que o diabetes tipo 1 é um fator de risco para o desenvolvimento de desordens psiquiátricas em crianças e adolescentes. As principais desordens são depressão, baixa autoestima e risco aumentado para as adolescentes de distúrbio alimentar. Os problemas de ajustamento ao diabetes podem aparecer logo após o diagnóstico da doença, mas muitas crianças resolvem este problema dentro de um ano, já as que não resolvem, começam a apresentar controle metabólico fraco, problemas de aderência ao regime e dificuldades psicossociais (Delamater \& cols., 2001).

O diabete é uma doença crônica, potencialmente invalidante, que determina mudanças internas nas atividades diárias da pessoa. São vivenciados vários sentimentos, como regressão, perda da auto-estima, insegurança, ansiedade, negação da situação apresentada e depressão. De acordo com a estrutura psíquica da pessoa e seus recursos internos, ela lidará, melhor ou pior, com a nova situação de doença... (Graça \& cols., 2000, p. 215)

Debray (1995) acredita que o diabetes será enfrentado diferentemente por cada indivíduo, pois dependerá da estrutura psíquica ou organização mental de cada um.

Segundo Pinkus (1988), o modo como o indivíduo enfrenta o diagnóstico da doença depende de três fatores:

1) o modo como soube da doença: se percebeu sozinho, se outro lhe mostrou, se adiou ter conhecimento do diagnóstico ou se preferiu saber logo.

2) as experiências pessoais anteriores que teve em relação a doença, por exemplo amigos ou pessoas da família que tiveram ou têm a doença.

3) o modo como a família e os amigos reagiram frente ao diagnóstico.

Ajuriaguerra (1976) parece concordar com Pinkus (1988) quando afirma que o enfrentamento da doença depende da história desta e da reação dos pais.

Zeidel (1970, citado em Joode, 1976, p. 23) considera que a criança diabética é mais susceptível a ter dificuldades de aprendizagem:

... há uma porcentagem maior de crianças emocionalmente perturbadas entre as crianças diabéticas do que na população em geral. Os problemas mais encontrados consistem principalmente de dificuldades no controle do diabetes, rebelião, depressão, distúrbio de comportamento e de aprendizagem. Quando surgem casos de neuroses e psicoses, a necessidade de tratamento psicoterápico tornase urgente, pois o diabetes tende a reforçar o desenvolvimento neurótico ou psicótico preexistente na criança ou na interação-familiar.

Num estudo comparativo Ajuriaguerra (1976) verificou que em crianças diabéticas, nas quais a doença se iniciou antes dos 5 anos de idade, o Q.I. apresentava diferenças significativas em relação as crianças nas quais a doença iniciou-se após os 5 anos de idade. Delamater e colaboradores (2001) também afirmam que problemas neurocognitivos em crianças e adolescentes podem estar relacionados tanto com história de controle glicêmico, quanto com a idade do início do diabetes (antes dos 5 anos de idade).
Joode (1976) pesquisou o Q.I. de diabéticos e constatou que $50 \%$ possuem Q.I. na faixa inferior à média e os outros $50 \%$ nas faixas média, superior à média e superior. Já Anjos (1982) mostra uma pesquisa realizada por uma psicóloga, a qual verificou o índice de inteligência de crianças diabéticas e concluiu que ele é $10 \%$ superior a das crianças não diabéticas. Vale e colaboradores (1979) também acreditam que as crianças diabéticas sejam psiquicamente mais vivas, mais alertas e mais maduras porque vivem uma doença complexa.

A família do diabético também enfrenta a ansiedade da perda frente a enfermidade que é dramática e exige cuidados, e por isso o diabetes deve ser tratado no grupo familiar, com um membro apoiando outro, pois através do apoio emocional os familiares tornam-se significativos no monitoramento do diabetes e na implementação das intervenções (Delamater, 2001; Soifer, 1982).

Joode (1976), em sua pesquisa, observou um alto índice de pais de diabéticos tipo 1, que apresentam muita ansiedade e esta se devia a problemas familiares ou conjugais, ao fato de não aceitarem a doença de seu filho e ao sentimento de culpa pelo fator hereditário envolvido na doença. Zanetti (1996), em sua pesquisa com 30 mães de pacientes diabéticos, afirma que $73,3 \%$ reagiu a doença com sentimento de revolta e desespero. Também Dylamater e colaboradores (2001) observaram sintomas de depressão significativos em um terço das mães de crianças diabéticas.

Debray (1995) faz uma análise da dinâmica familiar vivida pelo paciente diabético. Devido aos cuidados extremos exigidos pela doença os pais superprotegem os filhos, principalmente no caso de crianças, fazendo com que estas percam ou não adquiram autonomia. Ela também vê um aspecto inconsciente na superproteção; acredita que a superproteção pode estar encobrindo uma rejeição inconsciente. Foi observado, por ela, que esta rejeição e superproteção criaram uma falha narcísica em pacientes diabéticos.

... O filho portador de diabete não é a imagem que os pais planejaram para eles. É preciso fazer uma grande readaptação, trazendo o diabete para o plano que a família traçou para a criança. Os pais sentem-se ansiosos, culpados e tendem a superproteger a criança. Compensam, assim, a sobrecarga que sentem com o tratamento, as condutas e os procedimentos que precisam ser seguidos. É vivido um luto intenso pela criança sadia, que é perdida quando se formaliza o diabete. (Graça \& cols., 2000, p. 226)

Ajuriaguerra (1976, p. 838) mostra as características de crianças diabéticas e inclui a conseqüência da superproteção dos pais “... observa-se nessas crianças uma instabilidade de humor com irritabilidade, além de uma imaturidade afetiva que se traduz por grande necessidade de proteção, vontade imperiosa, falta de confiança em si e uma dependência prolongada em relação a um ou ambos genitores".

Joode (1976) e Anjos (1982) acreditam que a superproteção dos pais pode ajudar no controle da glicemia com sucesso, mas esmaga a personalidade da criança. Anjos cita o depoimento de uma diabética que viveu sobre a pressão esmagadora de sua mãe, que estava sempre limitando-a porque a considerava "a doente", no entanto a diabética sentia-se bem e conseguia conviver bem 
com a doença, com a dieta e com as injeções, mesmo quando criança. A paciente conta que chegou a comer muitos doces para descontrolar sua glicemia e então existir motivo para que sua mãe a considerasse uma doente.

Uma das maneiras de ajudar os pacientes diabéticos é através do trabalho proposto por Graça e colaboradores (2000), ou seja, integrar o diabético num grupo de iguais. O objetivo da formação de grupos é a melhora e não a cura, até mesmo porque é preciso pesar a realidade de que o diabetes mellitus é uma doença crônica e incurável até o presente momento. $\mathrm{O}$ falar sobre a doença possibilita trabalhar as fantasias dela, trocar entre iguais, compartilhar sentimentos, dúvidas e assim aprender a conviver melhor com ela.

Delamater e colaboradores (2001) também consideram importantíssimo o trabalho de grupo para jovens diabéticos, pois melhoram, a curto prazo, o controle glicêmico. Além disso atribuem importância às intervenções psicoeducacionais com crianças e suas famílias para resolver problemas e aumentar o apoio dos pais com relação a doença. Pesquisas têm demonstrado a eficácia de terapia psicossociais que podem melhorar a aderência ao regime, controle glicêmico, funcionamento psicossocial e qualidade de vida.

\section{Considerações Finais}

As pesquisas revelam que o diabetes está muito relacionado com o estado emocional dos seus portadores.

Esta relação entre o emocional e o diabetes existe antes mesmo do surgimento da doença, pois vários autores acreditam que o diabetes é um doença psicossomática, ou seja, que tem entre os seus fatores desencadeantes, causas emocionais. As causas mais comuns verificadas foram: traumas emocionais, modificações externas violentas, perda dos pais através de morte ou separação, problemas com os pais, com a familia e até com relação à escola.

Além da presença da relação entre o emocional e o diabetes tipo 1 na etiologia da doença, esta relação está também presente na conseqüência da doença. $\mathrm{O}$ diabetes pode provocar sentimentos de menos-valia, inferioridade, baixa auto-estima, medo, revolta, raiva, ansiedade, regressão, negação da doença, desesperança, incapacidade de amar e se relacionar bem com as pessoas, idéias de suicídio e depressão. A presença ou não destes sentimentos, dependerá dos recursos internos e da personalidade de cada um, da forma como foi dada a notícia da doença e como a família e os amigos reagiram frente ao diagnóstico.

O emocional também influenciará no controle da doença, uma vez que o diabetes exige um controle intenso para evitar complicações. Diante deste controle o paciente torna-se escravo do tratamento. O controle é uma questão ainda mais complicada para crianças e adolescentes, pois é preciso racionalização da doença e conseqüentemente do que se pode ou não fazer. Todavia a criança e o adolescente são bastante impulsivos, isto faz parte da característica de personalidade do ser humano nestas fases do desenvolvimento e é somente a partir da vida adulta que os desejos impulsivos são controlados em favor do racional. Assim torna-se extremamente difícil e estressante para as crianças e os adolescentes controlarem o diabetes, porque vivem de forma intensa uma ambivalência de sentimentos entre fazer aquilo que deseja e o que deve fazer.

Diante de toda a relação expressa entre o emocional e o diabetes observa-se que o acompanhamento psicológico a estes pacientes torna-se muito importante, pois proporcionará uma elaboração dos aspectos emocionais da doença e com isso minimizará os sofrimentos psíquicos. Para isso o psicólogo tem que observar, ouvir as palavras e os silêncios, vivenciar junto ao paciente os conflitos e problemas desta nova condição de ser diabético.

O trabalho psicológico com o paciente diabético pode realizar-se individualmente ou em grupo, ambos têm como objetivo a elaboração e aceitação da doença para obtenção de uma melhor qualidade de vida.

O trabalho psicoterapêutico individual pode ser realizado tanto com a criança ou o adolescente diabético, quanto com os pais afim de que eles também elaborem a perda do filho perfeito idealizado e aceitem a doença. Vale ressaltar a importância da orientação aos pais a respeito das características e repercussões da doença, para assim poderem ajudar seus filhos.

Com a contribuição do trabalho psicológico em grupo, as crianças e adolescentes diabéticos passam a não se sentirem sozinhos e a construir uma estrutura de personalidade não debilitada devido a doença.

Como todos sofrem de problemas semelhantes, enfrentam as mesmas vicissitudes e necessidades, há, no grupo, um forte nível de coesão e solidariedade. Uns se enxergam através dos outros. Os pacientes sentem-se protegidos e amparados. O grupo ajuda a combater a alienação, baixa auto-estima e desmoralização que ocorrem quando o indivíduo sente-se a única pessoa afligida pela doença. (Graça \& cols., 2000, pp. 216-217)

O trabalho em grupo é importante para que os pacientes busquem compartilhar sentimentos entre iguais e desfazer fantasias a respeito da doença, contribuindo assim, para uma aceitação interna dela e conseqüentemente uma melhor qualidade de vida.

\section{Referências}

Ajuriaguerra, J. de (1976). Manual de psiquiatria infantil ( $2^{\mathrm{a}}$ ed.). Rio de Janeiro: Masson do Brasil.

Anjos, M. N. dos (1982). A criança diabética ( $3^{\mathrm{a}}$ ed.). Rio de Janeiro: Cultura Médica. Brasil- Ministério da Saúde (2002). Vida saudável. Saúde do Brasil, dezembro. (Assessoria de Comunicação Social do Ministério da Saúde)

Debray, R. (1995). O equilibrio psicossomático: Um estudo sobre diabéticos. São Paulo: Casa do Psicólogo.

Delamater, A. M., Jacobson, A. M., Anderson, R. B., Cox, D., Fischer, L., Lustman, P., Rubin, R. \& Wisocki, T. (2001). Psychosocial therapies in diabetes. Diabetes Care, 24, 1286-1292.

Grupo de Estudos em Endocrinologia e Diabetes (2000). Diabetes- objetivando controle e educação. Endocrinologia \& Diabetes Clínica e Experimental, Curitiba, 1(3), 43-44.

Diabetes Mellitus (2003). Retirado em: 05/08/2003 no World Wide Web: http:// mnn:funasa.gov.br/pub/GVE/PDF/GVE0505.pdf

Diamond - Brasil, Ministério da Saúde (1993). Diabetes mellitus tipo 1- Incidência em alguns paises. Retirado em 24/07/2003 no World Wide Web: bttp:// dtr2001.saude.gov.br/sps/aresastecnicas/cnbd/publicacoes/bome.htm 
Gama, M. P. R. (2002). Do milagre canadense do século XX às esperanças de cura do século XXI (Editorial). Endocrinologia \& Diabetes Clínica e Experimental, 2(2), 3-5.

GEED - Grupo de Estudos em Endocrinologia \& Diabetes (2001). PROPOSTA de um estudo multicêntrico com diabéticos em uso de insulina. Endocrinologia \& Diabetes Clinica e Experimental (Curitiba), 1(5), 15-18.

Graça, L. A. C. da, Burd, M., Mello Fo., J. de (2000). Grupos com diabéticos. Em J. de Mello $F^{o}$. \& cols. (Orgs.), Grupo e corpo: Psicoterapia de grupo com pacientes somáticos (pp. 213-232). Porto Alegre: Artes Médicas.

Grünspun, H. (1980). Distúrbios psicossomáticos na criança. Rio de Janeiro: Atheneu. Hartmann, J. B., Dellatorre, A. \& Marcelino, D. B. (2002). Possibilidade de intervenção psicológica e resultados obtidos junto a pacientes diabéticos inseridos no Projeto Doce - Maringá. Endocrinologia \& Diabetes Clinica e Experimental (Curitiba), 2(2), 42-44.

Joode, M. S. de (1976). Influência dos fatores emocionais no diabetes mellitus. Dissertação de Mestrado não-publicada, Pontifícia Universidade Católica do Rio de Janeiro. Rio de Janeiro, RJ.

McCarty, \& Zimmet, (1994). Estimativas e Projeções Mundiais de DM no período de 1994-2010. Retirado em: 24/07/2003 no World Wibe Web: bttp:// dtr2001.saude.gov.br
Mello Fo , J. \& cols. (1992). Psicossomática hoje. Porto Alegre: Artes Médicas.

Musselman, D. L., Betan, E., Larsen, H. E. \& Phillips, L. S. (2003). Relationship of depression to diabetes types 1 and 2: Epidemiology, biology, and treatment. Biological Psychiatry, 54, 317-329.

Pinkus, L. (1988). Psicologia do doente. São Paulo: Paulinas.

Silva, M. A. D. da (1994). Quem ama não adoece. São Paulo: Best Seller.

Soifer, R. (1982). Psicodinamismo da família com crianças: Terapia familiar com técnica de jogo. Petrópolis, RJ: Vozes.

Vale, P. R. do, Ornellas, L. H. \& Franco, Z. (1979). Viva em paz com o seu diabetes (2a ed.). Rio de Janeiro: Atheneu.

Zagury, L., Zagury, T. \& Guidacci, J. (2000). Diabetes sem medo. Rio de Janeiro: Rocco. Zanetti, M. L. (1996). O diabetes mellitus tipo 1 em crianças e adolescentes: Um desafio para as mães e profissionais de saúde. Retirado em 15/06/2003 no World Wibe Web: bttp:// wnw.eerp.usp.br/aefund/ resumos/doutor_frame-1.htm.

Zimerman, D.\& Osório, L. C. (2000). Como trabalhamos com grupos. Porto Alegre: Artes Médicas.

Recebido: 26/08/2003

$1^{a}$ revisão: 04/12/2003

Aceite final: 22/01/2004

Sobre as autoras

Daniela Botti Marcelino é Psicóloga, e faz Mestrado na Universidade Estadual de Maringá.

Maria Dalva de Barros Carvalho é Pedagoga e Enfermeira. É Mestre pela Universidade Federal de São Carlos e Doutora pela Universidade de São Paulo. É Professora da Universidade Estadual de Londrina. 